\title{
Review Article \\ Tissue Engineering of Muscles and Cartilages Using Polyelectrolyte Hydrogels
}

\author{
Hyuck Joon Kwon \\ Department of Physical Therapy, College of Health Science, Eulji University, Gyeonggi 461-713, Republic of Korea \\ Correspondence should be addressed to Hyuck Joon Kwon; hyuckjoon5413@gmail.com
}

Received 22 September 2013; Accepted 17 March 2014; Published 25 June 2014

Academic Editor: Steven L. Suib

Copyright ( 2014 Hyuck Joon Kwon. This is an open access article distributed under the Creative Commons Attribution License, which permits unrestricted use, distribution, and reproduction in any medium, provided the original work is properly cited.

\begin{abstract}
The prevalent nature of osteoarthritis that causes the erosion of joint surfaces and loss of mobility and muscle dystrophy that weakens the musculoskeletal system and hampers locomotion underlies the importance of developing functional replacement or regeneration of muscle and cartilage tissues. Polyelectrolyte gels have high potential as cellular scaffolds due to characteristic properties similar to biological matrixes. A number of in vitro and in vivo studies demonstrated that polyelectrolyte gels are useful for replacement and regeneration of muscle and cartilage tissues. In addition, it was also found that polyelectrolyte gels have high biocompatibility, durability, and resistance to biodegradation. Moreover, polyelectrolyte gels can overcome their drawbacks of mechanical behavior by introducing double network into the gel. This paper reviews the current status and recent progress of polyelectrolyte gel-based tissue engineering for repairs of muscle and cartilage tissues.
\end{abstract}

\section{Introduction}

Impairment in skeletal muscle and cartilage tissues results from injury, aging, and disease such as osteoarthritis to result in the erosion of joint surfaces and loss of mobility and muscle dystrophy that weakens the musculoskeletal system and hampers locomotion $[1,2]$. Impaired function of muscle and cartilage influences the quality of life, preventing people from performing activities of daily living and being independent. Thus, replacement and regeneration of muscle and cartilage tissues are of great importance and interest not only scientifically but also clinically. However, despite the present knowledge of the biological processes underlying the regeneration of muscle and cartilage tissues, repair of the damaged tissues due to injury and pathological conditions remains a complex and unsolved task [3-5].

Hydrogels have long attracted considerable attention for tissue engineering because their hydrogels have the structural and compositional similarities to the biological tissues and their extensive framework for cellular proliferation and survival $[6,7]$. Although conventional hydrogels show no change in the equilibrium swelling with the change of the surrounding environment, polyelectrolyte gels which are a charged polymer network with macro ions fixed on the polymer chains and with micro counter-ions localized in the network frame show various unique responses to environmental stimuli such as electrically induced chemomechanical contraction similar to biological responses [810]. Furthermore, biological tissues consist of polyelectrolytes such as polysaccharide and charged filamentous proteins and their properties originate from the polyelectrolyte nature [11-13]. Therefore, polyelectrolyte gels have shown considerable potential as excellent scaffolds for cellular adhesion, proliferation, and differentiation and as artificial tissues for replacement of damaged tissues [14].

This review article describes biomedical application of polyelectrolyte gels for replacement and regeneration of muscle and cartilage tissues.

\section{Development of an Artificial Muscle Using Polyelectrolyte Gel}

Hydrogel composed of polyelectrolytes can produce the motion by the chemical free energy of the polymer network. This suggests that polyelectrolyte gel can be used as a chemomechanical actuator to produce a flexible action and a soft-actuator like muscle movement. It was demonstrated 


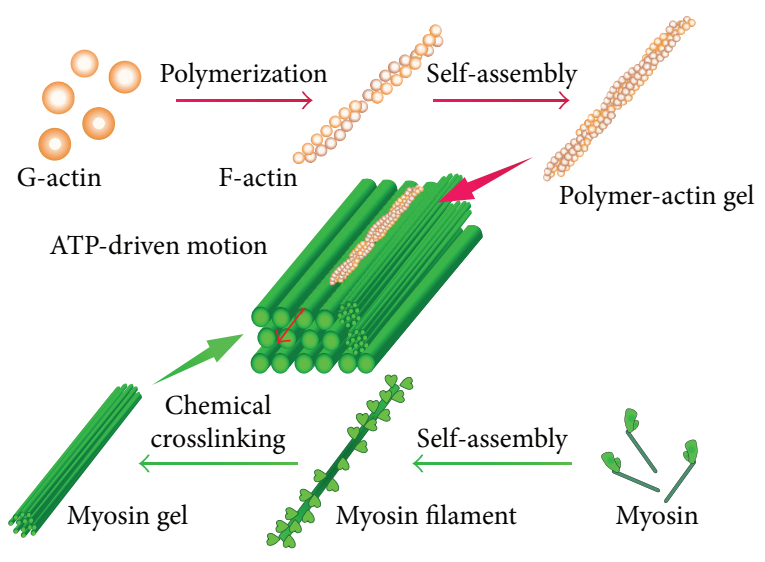

(a)
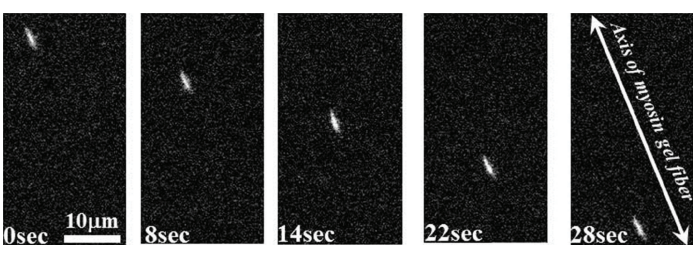

(b)

Figure 1: Scheme (a) and fluorescence images (b) of the motion of the ATP fueled actin-polymer gel actuator on the oriented myosin gel. Reprinted with permission from [20] () 2002, John Wiley \& Sons, Inc.

that the negatively charged PAMPS gel moved by reversible and cooperative complexation by an electrical stimulation $[15,16]$. In the gel actuator, the electric field gave anisotropic complex formation by controlling the direction of this equilibrium. While the DC voltage was turned on, the positively charged surfactant molecules moved towards the cathode, bound to the negatively charged gel preferentially on the side of the PAMPS facing the anode. This consequently led to anisotropic contraction by bending the gel towards the anode. The reverse direction of the electric field induced the surfactant molecules bound to the gel to be released and moved towards the anode and new surfactant molecules to bind preferentially to the opposite side of the gel and straightened the gel. Consequently, the polyelectrolyte gel can bend and stretch repeatedly by controlling electrical field.

It was also shown that negatively charged polyacrylonitrile (PAN) gel can be used as a gel actuator $[17,18]$. While PAN gel immersed repeatedly in caustic and acidic solutions, the PAN gel elongates and contracts repeatedly. The strength of PAN gels was comparable to that of human muscle. Moreover, although PAN gel had the drawback of the need of strong acids and bases for actuation, it was reported that increasing the conductivity by depositing platinum on the PAN fibers or combining the PAN fibers with graphite fibers induced electrical activation of the PAN gel [19]. This result indicates that PAN gel can be used as an artificial muscle thorough electrical control.

In addition, to overcome the lack of hierarchical structures and biological energy sources inside the gel composed of synthetic polymers, the biopolymer gel actuator was made from negatively charged actin filaments and polycations [20]. The actin-polymer complex gel was reported to move along a myosin fibrous gel by using ATP energy (Figure 1). This result indicates that the actin-polymer complex gel can be used as an ATP-fueled artificial muscle. This biopolymerbased actuator might operate in human body as an actuator without causing any immunoreactions.

\section{Development of an Artificial Cartilage Using Polyelectrolyte Gel}

Function repair of an articular cartilage defect remains a major challenge in the field of joint surgery because it is extremely difficult to regenerate these tissues with currently available therapeutic treatments. Therefore, it is important to develop biomaterials to replace the normal cartilage tissue $[24,25]$. Although hydrogels have a considerable potential as scaffolds for tissue engineering due to their high biocompatibility, the use of hydrogel as an artificial cartilage has been severely limited by their weak mechanical behavior. However, it was demonstrated that polyelectrolyte gel can have high fracture energy and dynamic stiffness close to native cartilage by introducing the neutral polymers into polyelectrolyte networks (Figure 2) $[26,27]$. The double network gel composed of PAMPS and PDMAAm (PAMPS/PDMAAm DN gel) had a wear property comparable to the clinically available ultra-high molecular weight polyethylene [27, 28]. Moreover, PAMPS/PDMAAm DN gel caused a mild inflammation at 1 week after the gel implantation, and the inflammation degree significantly decreased at 4 and 6 weeks [29], and the implantation of PAMPS/PDMAAm DN gel into a femoral cartilage defect induced no significant damage to the opposite normal cartilage in the patella as well as to the postoperative healing of the knee joint [30]. There were no statistical differences in the cartilage surface roughness and the number of the small pits between the DN gel and the normal cartilage surface and the coefficient of the DN gel to normal cartilage articulation was significantly lower than that of the normal-to-normal cartilage articulation [30]. Recently, high stretchable and tough polyelectrolyte-based gel was developed by double network structure of negatively charged alginate and neutral polyacrylamide [31]. It was demonstrated that the gel can be stretched beyond 20 times its initial length and has fracture energies of about $9000 \mathrm{Jm}^{-2}$. It was suggested that the toughness of the double network gel is due to the synergy mechanism of cracking bridging by the network of covalent crosslinks and hysteresis by unzipping the network 


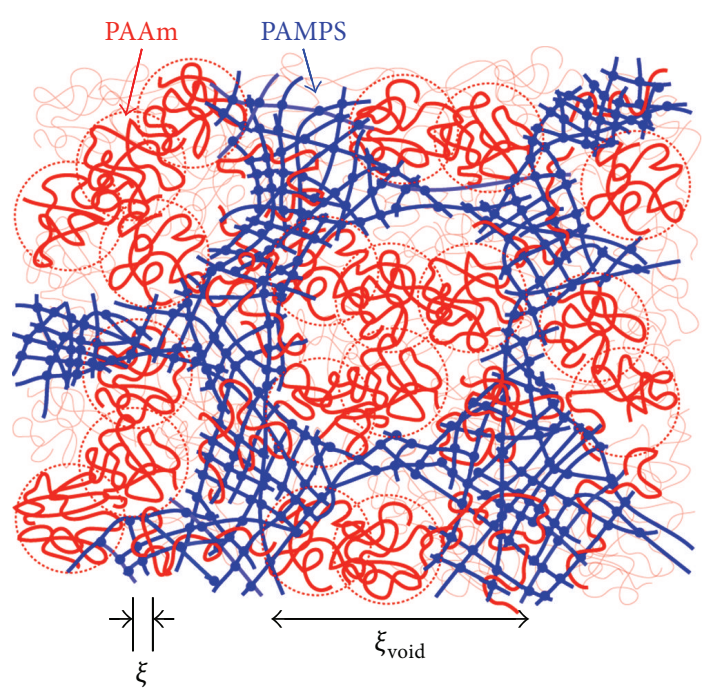

FIGURE 2: Inhomogeneous structure model for the tough DN gels. The first network PAMPS has a mesh size $\xi$ of several nm with large "voids" of size $\xi_{\text {void }} \gg \xi$. The second component PAAm chains are in concentrated region and they both are entangled with each other and with the PAMPS network. For clarity, only some of the PAAm chains are highlighted by bolder lines. The voids that are filled with concentrated PAAm act as "crack-stop" to enhance the mechanical strength of the DN gels. Reproduced with permission from [14] (C) 2003, Elsevier, Inc.

of polyelectrolyte crosslinks. These studies suggest that the polyelectrolyte gel can be a promising material to replace the artificial cartilage.

\section{Muscle Regeneration Using Polyelectrolyte Gel}

Although hydrogels are useful for cell scaffolds [32, 33], various cell-interacting components such as ECM molecules, small peptide, and glycoproteins need to be incorporated into hydrogels for cell attachment [34-38]. However, it was found that negatively charged hydrogels, such as PAMPS, poly(2-acrylamido-2-methylpropane sulfonic acid sodium salt) (PNaAMPS), and poly (sodium p-styrene sulfonate) (PNaSS), are suitable scaffolds for cell culture without any modification of cell adhesive protein (Figure 3) [21, 39-41]. These results suggest that polyelectrolyte gel can be a useful cell scaffold.

Despite much knowledge of biological processes underlying muscle regeneration, reconstruction of skeletal muscle due to injury or pathological conditions remains an unresolved task [42]. When muscle progenitor cells (MPCs) were injected into the damaged tissue, MPCs rapidly lost their myogenic potential on in vitro expansion and displayed high mortality [43]. However, it was reported that when MPCs were delivered via the negatively charged hyaluronan-based hydrogel, the reconstruction of skeletal muscle was promoted histologically and functionally [44]. Additionally, Kamelger et al. showed that myoblasts seeded in negatively charged hydrogels such as alginate and hyaluronic acid-hydrogels can fuse in vivo into vascularized and multinucleated myotubes [45].

\section{Cartilage Regeneration Using Polyelectrolyte Gel}

Our study showed that the adhesion and the proliferation of chondrocyte progenitor cells increased with charge density of negatively charged PAMPS gel [22]. Moreover, it was demonstrated that the highly charged PAMPS gel promoted chondrogenic differentiation even in the absence of differentiation supplements and the chondrogenic differentiation degree increased with charge density of PAMPS gel (Figure 4) [22]. Since PAMPS has similarities with proteoglycans in its negative charge and sulfonic acid base, it was suggested that PAMPS gel plays key roles for chondrogenic differentiation like proteoglycans which directly function as a signal molecule to interact with receptors for signaling pathways and act as an effective reservoir for signaling molecules or growth factors.

In addition, when it was examined how the mechanical property of hydrogel coated with negatively charged sulfonate groups influences differentiation of mesenchymal stem cell (MSC), it was shown that MSC chondrogenesis depended on the stiffness of the negatively charged hydrogels and that the soft polyelectrolyte gel effectively induced MSC chondrogenesis even in the absence of differentiation supplements, in comparison with the rigid polyelectrolyte gels (Figure 5) [23]. This result implicates that polyelectrolyte gel-induced chondrogenesis depends on the gel stiffness.

In addition to in vitro study, PAMPS gel was shown to induce in vivo cartilage regeneration in a large osteochondral defect of the rabbit [46]. Moreover, the PAMPS-based double network gel which can be clinically available due to high mechanical strength also induced cartilage regeneration in vivo at 4 weeks after implantation of DN gel into the defect [47]. The DN gel-implanted defect was almost completely filled with the hyaline cartilage tissue rich in chondrogenic markers such as proteoglycan and type 2 collagen [48]. It was also found that the expression level of chondrogenic marker type II collagen in the regenerated tissue was obviously higher in the DN gel-implanted defect than in the untreated control. The surface roughness of the untreated control was significantly higher than the normal cartilage at 12 weeks, while there was no statistical difference between the DN gel-implanted and normal knees. This induction of cartilage regeneration by the polyelectrolyte gel can provide a novel strategy to repair an osteochondral defect in the field of joint surgery. This novel gel strategy needs to be further validated in a larger animal model to determine its efficacy for possible use in the human.

\section{Evaluation of Polyelectrolyte Gel for Practical Use for Tissue Engineering}

Potential materials for practical use in replacement and regeneration of biological tissues are required to be biocompatible, durable to mechanical stresses, resistant to wear, 

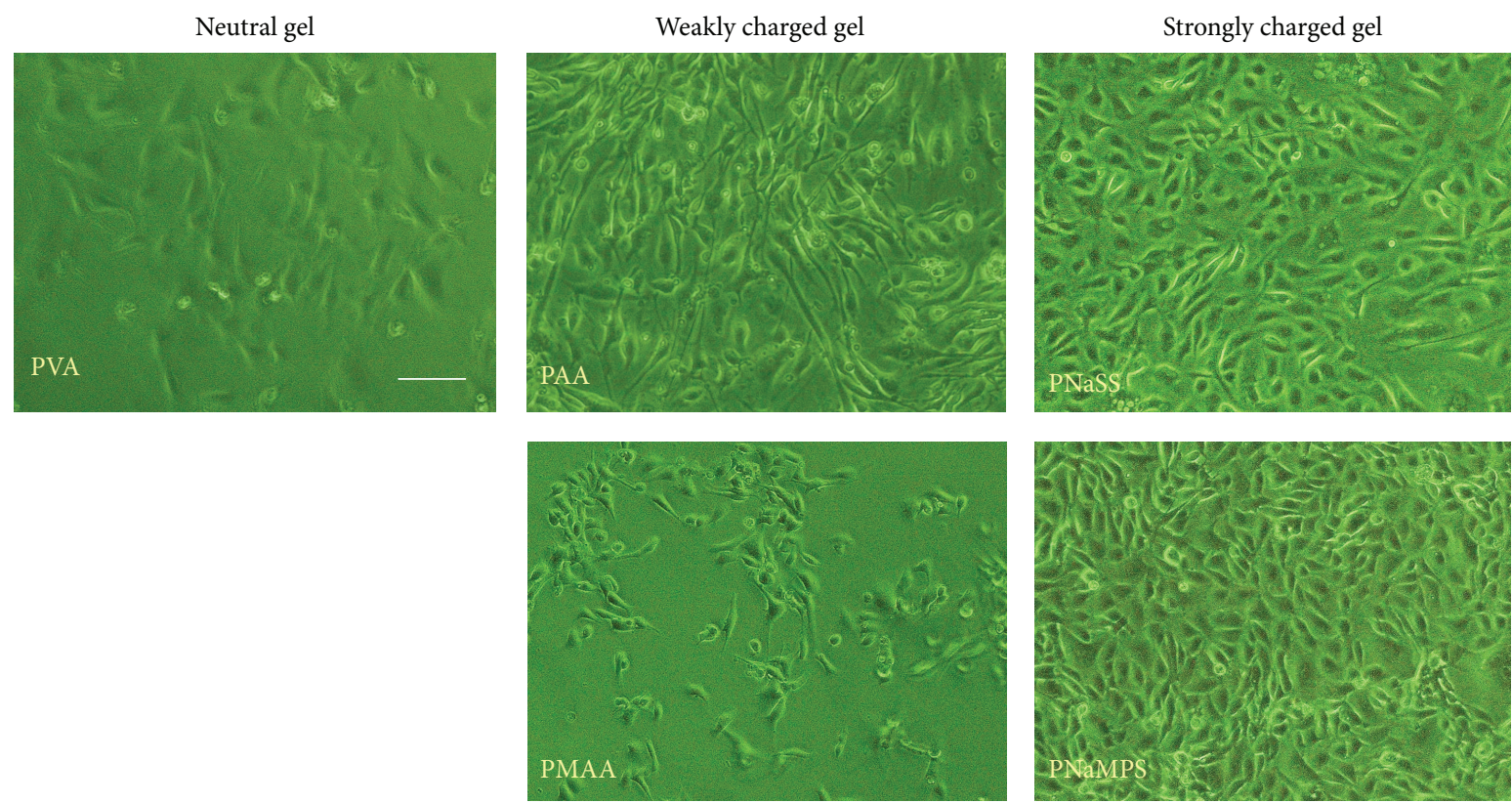

FIGURE 3: Phase-contrast micrographs of bovine fetal aorta endothelial cell (BFAECs) on various gels at a prolonged culture time (120 h). Crosslinker concentration of gels: PVA (6 mol\%), PAA (2 mol\%), PMAA (1 mol\%), PNaSS (10 mol\%), and PNaAMPS (6 mol\%). Original magnification: $\times 10$. Scale bar: $100 \mu \mathrm{m}$. Reproduced with permission from [21]. ๑ 2003, Elsevier, Inc.
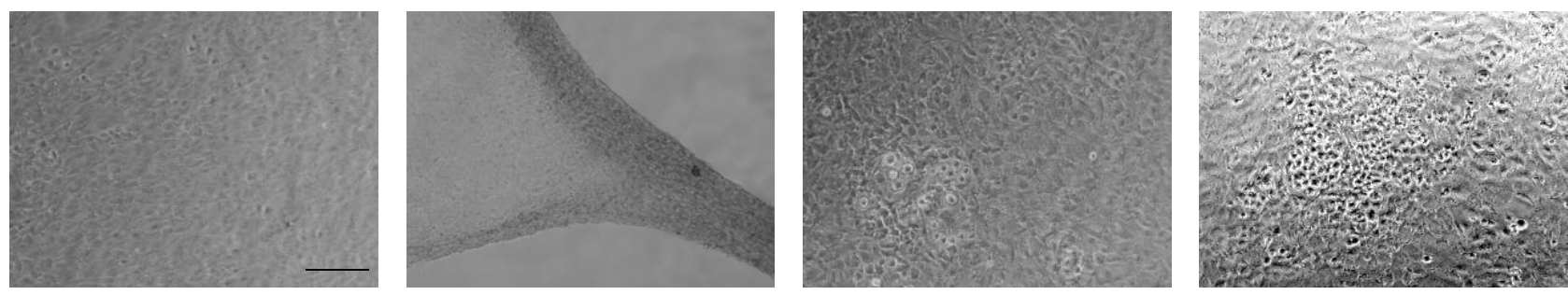

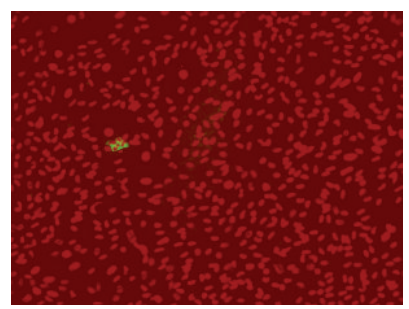

PS

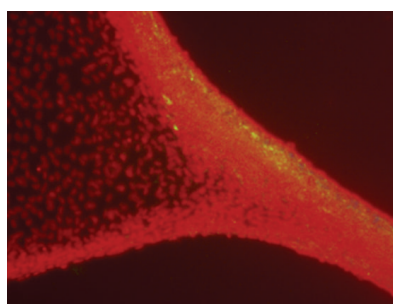

P(AMPS-CO-DMAAm) $(F=0.5)$

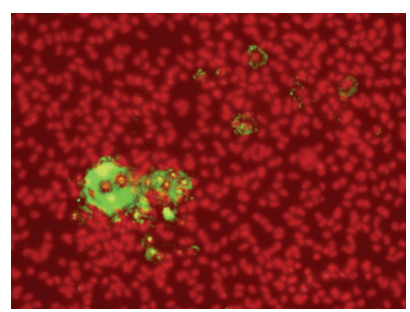

$\mathrm{P}$ (AMPS-CO-DMAAm) $(F=0.75)$

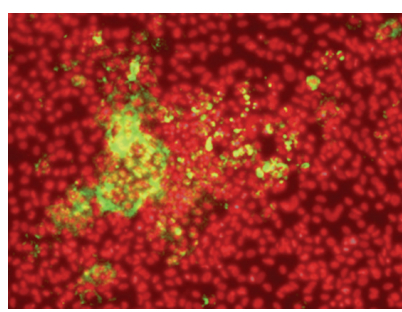

PAMPS

FIGURE 4: Expression of chondrogenic markers in ATDC5 cells cultured on hydrogels with various charge density; Polystyrene dish (PS), PDMAAm gel, P(AMPS-co-DMAAm) (molar fraction of AMPS, $F=0.5,0.75$ ), and PAMPS gel, in the absence of insulin. Scale bar, $100 \mu \mathrm{m}$. Expression of type II collagen was analyzed by immunofluorescent staining at 14 days of culture. The cells were stained with an antitype II collagen antibody (green) and Hoechst 33258 (red). Reproduced with permission from [22]. (c) 2010, Elsevier, Inc.

and resistant to biodegradation. It was found that synthetic polyelectrolyte gels such as PAMPS, PNaAMPS, and PNaSS did not have cytotoxicity in vitro $[21,40]$. In addition, since PAMPS gel has a high potential for not only muscle and cartilage replacement but also cartilage regeneration, the biological reactions of PAMPS-based hydrogel were evaluated in vivo by implantation of the gel into a subcutaneous tissue [27-29]. The result showed that PAMPS-based hydrogel induced a mild inflammation at 1 week, but the degree of the inflammation significantly decreased at 4 and 6 weeks into the same degree as that of negative control [29]. Moreover, it was demonstrated that when the implanted gel specimens were carefully harvested from the subcutaneous tissue and then the harvested gel specimens underwent pin-on-flat-type wear testing, the gel revealed an excellent wear property [27]. It was also found that the PAMPS-based gel was hardly degraded 


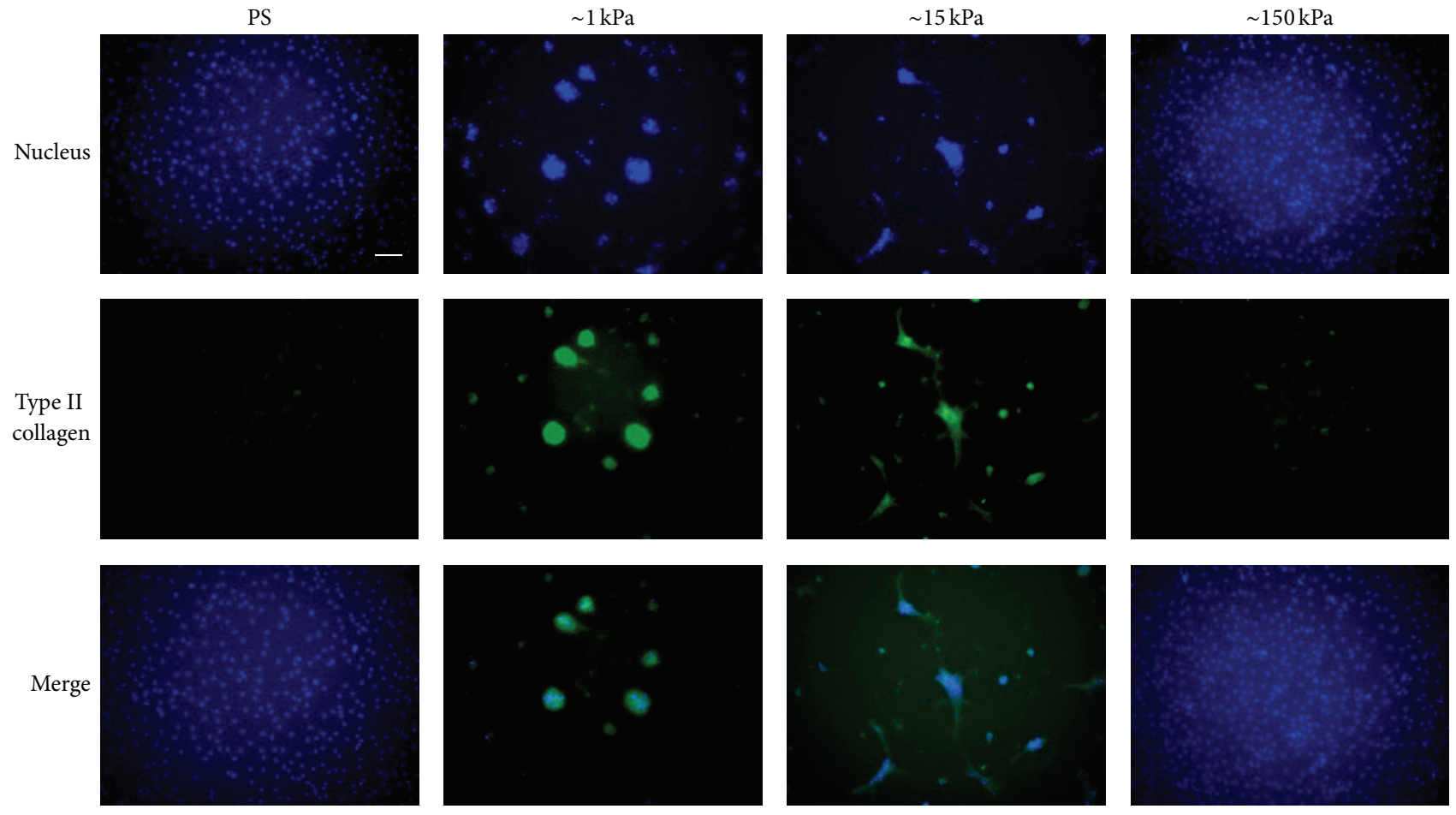

FIGURE 5: Expression levels of type II collagen in MSCs after 1 week culture on polystyrene dish (PS), S-PAAm gel with $E$ of $\sim 1, \sim 15$, and $\sim 150 \mathrm{kPa}$, respectively. Scale bar, $100 \mu \mathrm{m}$. Type II collagen was detected with immunofluorescent. In the immunofluorescent staining, the cells were stained with an antitype II collagen antibody (green) and Hoechst 33258 (blue). Reproduced with permission from [23]. @ 2010, Elsevier, Inc.

when it was implanted into living animals [28]. These results indicate that PAMPS gel is a practically useful biomaterial for tissue engineering.

\section{Conclusion}

A loss of muscle and cartilage tissues has resulted in the functional impairment and cosmetic deformation of patients. Tissue engineering of muscle and cartilage are growing fields of interest in aged society. Since polyelectrolyte gels have high biocompatibility and chemical properties similar to biological matrixes and moreover polyelectrolyte gels can obtain high strength as high as a few to several tens of megapascals and high stretchability by incorporating double network structure into the gel. A number of studies demonstrated that polyelectrolyte gels are useful biomaterials to substitute for muscle and cartilage tissues and have potential properties for regeneration of muscle and cartilage tissues.

Therefore, polyelectrolyte gels have potential for many applications in tissue engineering and regenerative medicine. However, there are still major hurdles to overcome in clinical applications. The potential applications and benefits for reconstructive surgery will grow with the development of more sophisticated techniques in tissue engineering science.

\section{Conflict of Interests}

The author declares no conflict of interests.

\section{Acknowledgment}

This paper was supported by Eulji University in 2013.

\section{References}

[1] R. Stockwell, Biology of Cartilage Cells, Cambridge University Press, Cambridge, UK, 1979.

[2] S. V. Brooks, "Current topics for teaching skeletal muscle physiology," Advances in Physiology Education, vol. 27, no. 1-4, pp. 171-182, 2003.

[3] J. D. Rosenblatt, A. I. Lunt, D. J. Parry, and T. A. Partridge, "Culturing satellite cells from living single muscle fiber explants," In Vitro Cellular and Developmental Biology-Animal, vol. 31, no. 10, pp. 773-779, 1995.

[4] B. R. Mandelbaum, J. E. Browne, F. Fu et al., "Articular cartilage lesions of the knee," The American Journal of Sports Medicine, vol. 26, no. 6, pp. 853-861, 1998.

[5] J. A. Buckwalter and H. A. Mankin, "Articular cartilage repair and transplantation," Arthritis Rheumatism, vol. 41, no. 8, pp. 1331-1342, 1998.

[6] N. A. Peppas and R. Langer, "New challenges in biomaterials," Science, vol. 263, no. 5154, pp. 1715-1720, 1994.

[7] N. A. Peppas, Hydrogels in Medicine and Pharmacy, CRC Press, Boca Raton, Fla, USA, 1987.

[8] T. Tanaka, I. Nishio, S. T. Sun, and S. Ueno-Nishio, "Collapse of gels in an electric field," Science, vol. 218, no. 4571, pp. 467-469, 1982. 
[9] Y. Osada and J. P. Gong, "Stimuli-responsive polymer gels and their application to chemomechanical systems," Progress in Polymer Science, vol. 18, no. 2, pp. 187-226, 1993.

[10] R. Yoshida, "Self-oscillating gels driven by the belousovzhabotinsky reaction as novel smart materials," Advanced Materials, vol. 22, no. 31, pp. 3463-3483, 2010.

[11] W. D. Comper and T. C. Laurent, "Physiological function of connective tissue polysaccharides," Physiological Reviews, vol. 58, no. 1, pp. 255-315, 1978.

[12] J. X. Tang and P. A. Janmey, "The polyelectrolyte nature of F-actin and the mechanism of actin bundle formation," The Journal of Biological Chemistry, vol. 271, no. 15, pp. 8556-8563, 1996.

[13] G. C. Wong and L. Pollack, "Electrostatics of strongly charged biological polymers: ion-mediated interactions and selforganization in nucleic acids and proteins," Annual Review of Physical Chemistry, vol. 61, pp. 171-189, 2010.

[14] H. J. Kwon and J. P. Gong, "Negatively charged polyelectrolyte gels as bio-tissue model system and for biomedical application," Current Opinion in Colloid and Interface Science, vol. 11, no. 6, pp. 345-350, 2006.

[15] Y. Osada, H. Okuzaki, and H. Hori, "A polymer gel with electrically driven motility," Nature, vol. 355, no. 6357, pp. 242244, 1992.

[16] Y. Osada and S. Ross-Murphy, "Intelligent gels," Scientific American, vol. 268, pp. 82-87, 1993.

[17] D. Y. Lee, Y. Kim, S. J. Lee et al., "Characteristics of chemomechanically driven polyacrylonitrile fiber gel actuators," Materials Science and Engineering C, vol. 28, no. 2, pp. 294-298, 2008.

[18] K. Choe and K. J. Kim, "Polyacrylonitrile linear actuators: chemomechanical and electro-chemomechanical properties," Sensors and Actuators A: Physical, vol. 126, no. 1, pp. 165-172, 2006.

[19] H. B. Schreyer, N. Gebhart, K. J. Kim, and M. Shahinpoor, "Electrical activation of artificial muscles containing polyacrylonitrile gel fibers," Biomacromolecules, vol. 1, no. 4, pp. 642-647, 2000.

[20] A. Kakugo, S. Sugimoto, J. P. Gong, and Y. Osada, "Gel machines constructed from chemically cross-linked actins and myosins," Advanced Materials, vol. 14, no. 16, pp. 1124-1126, 2002.

[21] Y. M. Chen, N. Shiraishi, H. Satokawa et al., "Cultivation of endothelial cells on adhesive protein-free synthetic polymer gels," Biomaterials, vol. 26, no. 22, pp. 4588-4596, 2005.

[22] H. J. Kwon, K. Yasuda, Y. Ohmiya, K. Honma, Y. M. Chen, and J. P. Gong, "In vitro differentiation of chondrogenic ATDC5 cells is enhanced by culturing on synthetic hydrogels with various charge densities," Acta Biomaterialia, vol. 6, no. 2, pp. 494-501, 2010.

[23] H. J. Kwon, "Chondrogenesis on sulfonate-coated hydrogels is regulated by their mechanical properties," Journal of the Mechanical Behavior of Biomedical Materials, vol. 17, pp. 337346, 2012.

[24] K. E. M. Benders, P. R. van Weeren, S. F. Badylak, D. B. Saris, W. J. Dhert, and J. Malda, "Extracellular matrix scaffolds for cartilage and bone regeneration," Trends in Biotechnology, vol. 31, no. 3, pp. 169-176, 2013.

[25] P. Ducheyne, R. L. Mauck, and D. H. Smith, "Biomaterials in the repair of sports injuries," Nature Materials, vol. 11, no. 8, pp. 652-654, 2012.

[26] J. P. Gong, Y. Katsuyama, T. Kurokawa, and Y. Osada, "Doublenetwork hydrogels with extremely high mechanical strength," Advanced Materials, vol. 15, no. 14, pp. 1155-1158, 2003.
[27] K. Yasuda, J. P. Gong, Y. Katsuyama et al., "Biomechanical properties of high-toughness double network hydrogels," Biomaterials, vol. 26, no. 21, pp. 4468-4475, 2005.

[28] C. Azuma, K. Yasuda, Y. Tanabe et al., "Biodegradation of hightoughness double network hydrogels as potential materials for artificial cartilage," Journal of Biomedical Materials Research A, vol. 81, no. 2, pp. 373-380, 2007.

[29] Y. Tanabe, K. Yasuda, C. Azuma et al., "Biological responses of novel high-toughness double network hydrogels in muscle and the subcutaneous tissues," Journal of Materials Science: Materials in Medicine, vol. 19, no. 3, pp. 1379-1387, 2008.

[30] K. Arakaki, N. Kitamura, H. Fujiki et al., "Artificial cartilage made from a novel double-network hydrogel: in vivo effects on the normal cartilage and ex vivo evaluation of the friction property," Journal of Biomedical Materials Research A, vol. 93, no. 3, pp. 1160-1168, 2010.

[31] J. Y. Sun, X. Zhao, W. R. Illeperuma et al., "Highly stretchable and tough hydrogels," Nature, vol. 489, no. 7414, pp. 133-136, 2012.

[32] N. S. Hwang, S. Varghese, and J. Elisseeff, "Controlled differentiation of stem cells," Advanced Drug Delivery Reviews, vol. 60, no. 2, pp. 199-214, 2008.

[33] H. J. Lee, J. S. Lee, T. Chansakul, C. Yu, J. H. Elisseeff, and S. M. $\mathrm{Yu}$, "Collagen mimetic peptide-conjugated photopolymerizable PEG hydrogel," Biomaterials, vol. 27, no. 30, pp. 5268-5276, 2006.

[34] N. S. Hwang, S. Varghese, Z. Zhang, and J. Elisseeff, "Chondrogenic differentiation of human embryonic stem cell-derived cells in arginine-glycine-aspartate-modified hydrogels," Tissue Engineering, vol. 12, no. 9, pp. 2695-2706, 2006.

[35] L. M. Weber, K. N. Hayda, K. Haskins, and K. S. Anseth, "The effects of cell-matrix interactions on encapsulated $\beta$-cell function within hydrogels functionalized with matrix-derived adhesive peptides," Biomaterials, vol. 28, no. 19, pp. 3004-3011, 2007.

[36] G. D. Nicodemus and S. J. Bryant, "The role of hydrogel structure and dynamic loading on chondrocyte gene expression and matrix formation," Journal of Biomechanics, vol. 41, no. 7, pp. 1528-1536, 2008.

[37] A. J. Engler, S. Sen, H. L. Sweeney, and D. E. Discher, "Matrix elasticity directs stem cell lineage specification," Cell, vol. 126, no. 4, pp. 677-689, 2006.

[38] N. E. Fedorovich, J. Alblas, J. R. de Wijn, W. E. Hennink, A. J. Verbout, and W. J. Dhert, "Hydrogels as extracellular matrices for skeletal tissue engineering: state-of-the-art and novel application in organ printing," Tissue Engineering, vol. 13, no. 8, pp. 1905-1925, 2007.

[39] Y. M. Chen, R. Ogawa, A. Kakugo, Y. Osada, and J. P. Gong, "Dynamic cell behavior on synthetic hydrogels with different charge densities," Soft Matter, vol. 5, no. 9, pp. 1804-1811, 2009.

[40] Y. M. Chen, J. P. Gong, M. Tanaka et al., "Tuning of cell proliferation on tough gels by critical charge effect," Journal of Biomedical Materials Research A, vol. 88, no. 1, pp. 74-83, 2009.

[41] Y. M. Chen, J. J. Yang, and J. P. Gong, "Adhesion, spreading, and proliferation of endothelial cells on charged hydrogels," Journal of Adhesion, vol. 85, no. 11, pp. 839-868, 2009.

[42] E. L. Fong, C. K. Chan, and S. B. Goodman, "Stem cell homing in musculoskeletal injury," Biomaterials, vol. 32, no. 2, pp. 395409, 2010. 
[43] J. R. Beauchamp, J. E. Morgan, C. N. Pagel, and T. A. Partridge, "Dynamics of myoblast transplantation reveal a discrete minority of precursors with stem cell-like properties as the myogenic source," Journal of Cell Biology, vol. 144, no. 6, pp. 1113-1121, 1999.

[44] C. A. Rossi, M. Flaibani, B. Blaauw et al., "In vivo tissue engineering of functional skeletal muscle by freshly isolated satellite cells embedded in a photopolymerizable hydrogel," The FASEB Journal, vol. 25, no. 7, pp. 2296-2304, 2011.

[45] F. S. Kamelger, R. Marksteiner, E. Margreiter et al., "A comparative study of three different biomaterials in the engineering of skeletal muscle using a rat animal model," Biomaterials, vol. 25, no. 9, pp. 1649-1655, 2004.

[46] M. Ogawa, N. Kitamura, T. Kurokawa et al., "Poly(2-acrylamido-2-methylpropanesulfonic acid) gel induces articular cartilage regeneration in vivo: comparisons of the induction ability between single- and double-network gels," Journal of Biomedical Materials Research A, vol. 100, no. 9, pp. 2244-2251, 2012.

[47] K. Yasuda, N. Kitamura, J. P. Gong et al., "A novel doublenetwork hydrogel induces spontaneous articular cartilage regeneration in vivo in a large osteochondral defect," Macromolecular Bioscience, vol. 9, no. 4, pp. 307-316, 2009.

[48] N. Kitamura, K. Yasuda, M. Ogawa et al., "Induction of spontaneous hyaline cartilage regeneration using a double-network gel: efficacy of a novel therapeutic strategy for an articular cartilage defect," The American Journal of Sports Medicine, vol. 39, no. 6, pp. 1160-1169, 2011. 

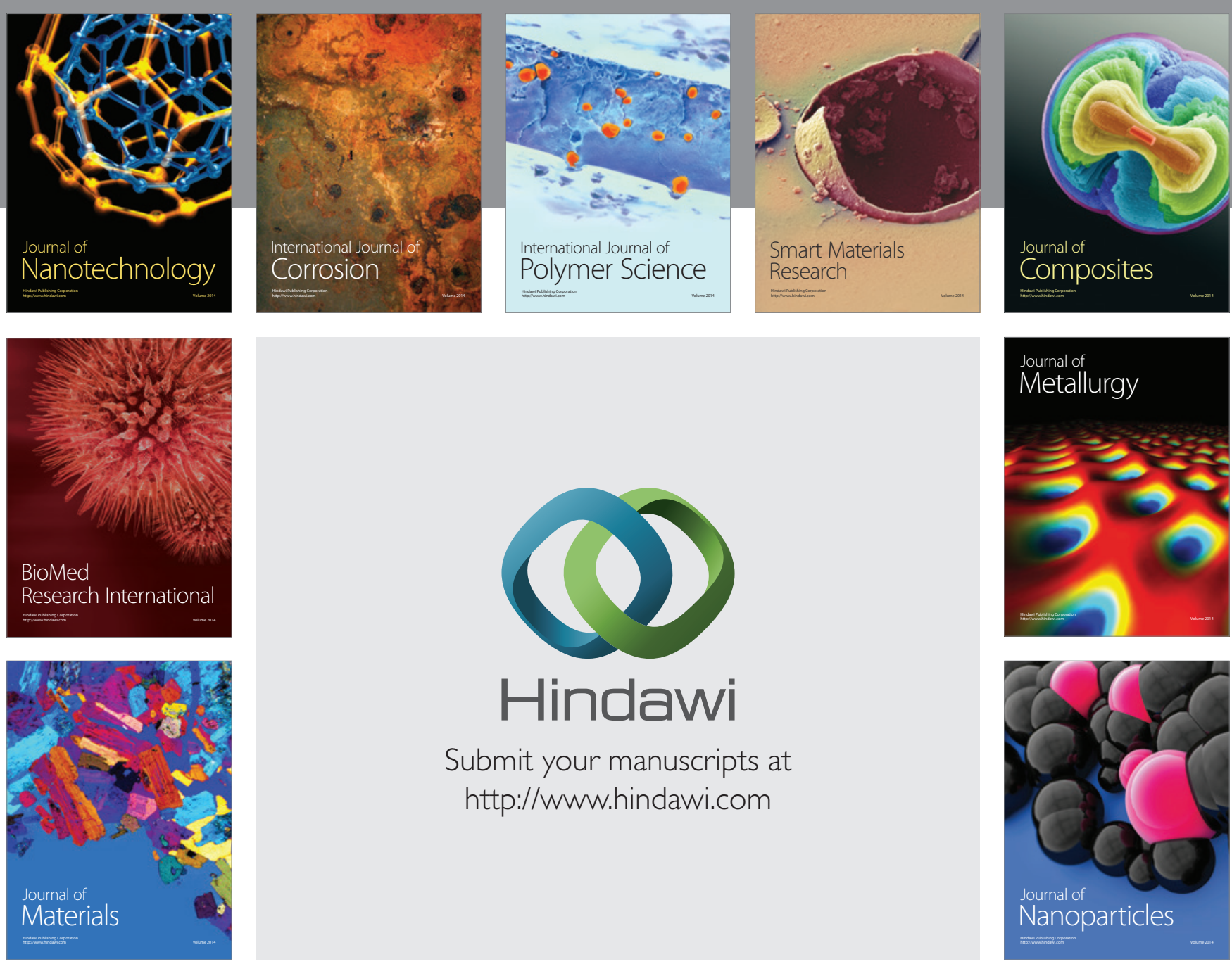

Submit your manuscripts at http://www.hindawi.com
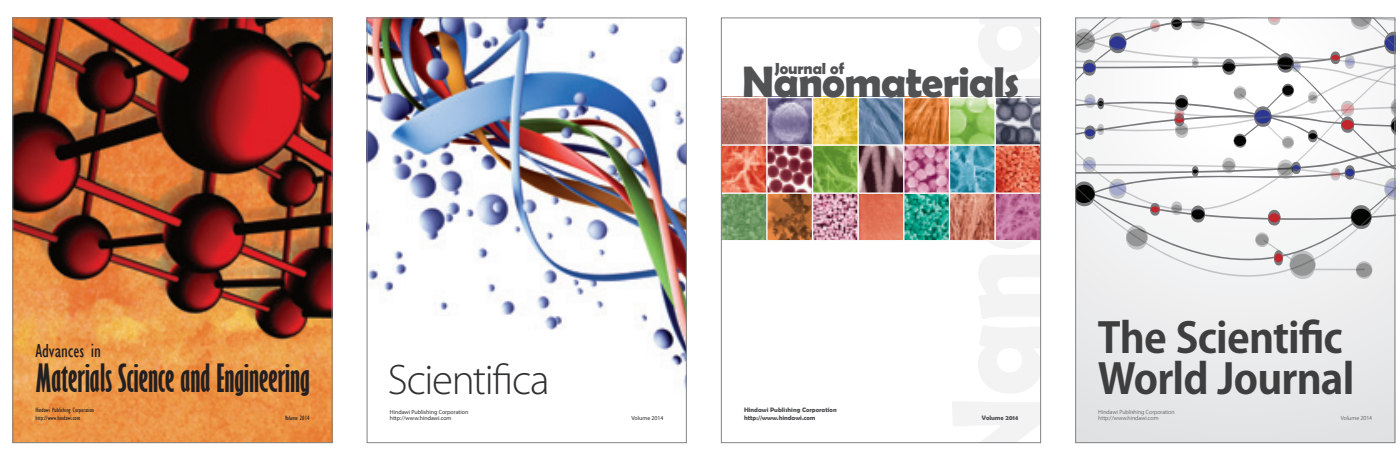

\section{The Scientific World Journal}
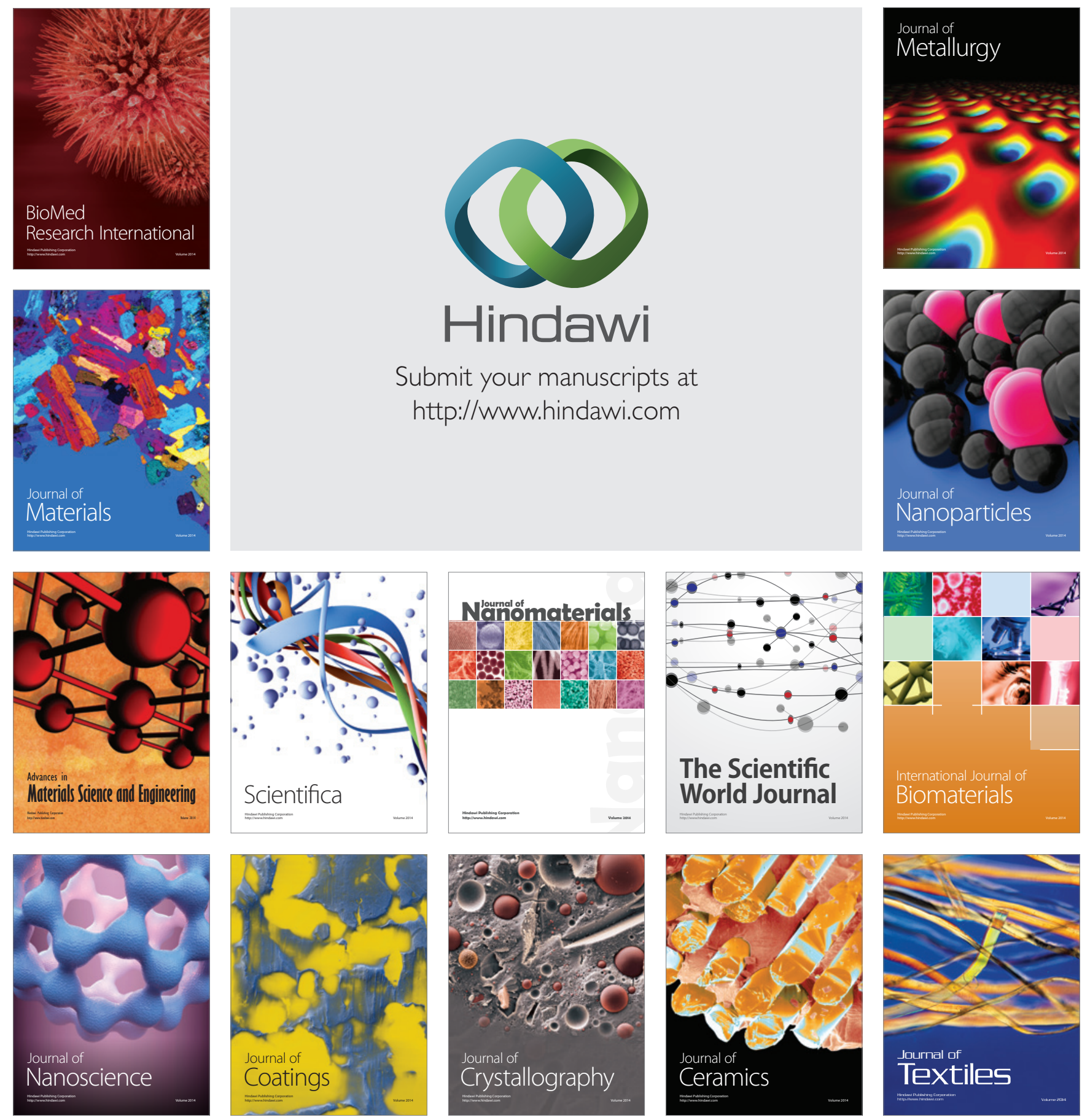